\title{
Vitamin D Insufficiency and Cognitive Function Trajectories in Older Adults: The Rancho Bernardo Study
}

\author{
Gail A Laughlin, PhD ${ }^{\mathrm{a}}$, Donna Kritz-Silverstein, PhD $^{\mathrm{a}}$, Jaclyn Bergstrom, MS ${ }^{\mathrm{a}}$, Emilie T. \\ Reas, $\mathrm{PhD}^{\mathrm{b}}$, Simerjot K. Jassal, MD $^{\mathrm{c}}$, Elizabeth Barrett-Connor, $\mathbf{M D}^{\mathrm{a}}$, and Linda K. McEvoy, \\ $\mathrm{PhD}^{\mathrm{a}, \mathrm{b}}$ \\ aDivision of Epidemiology, Department of Family Medicine and Public Health, University of \\ California San Diego

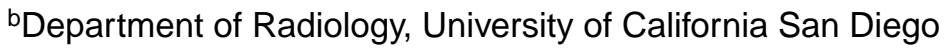 \\ 'Division of General Internal Medicine, Department of Medicine, VA San Diego Healthcare \\ System, University of California San Diego
}

\begin{abstract}
Background-Evidence of a role for vitamin D (VitD) in cognitive aging is mixed and based primarily on extreme VitD deficiency. We evaluated the association of VitD insufficiency with cognitive function in older, community-dwelling adults living in a temperate climate with year round sunshine.
\end{abstract}

\begin{abstract}
Methods-A population-based longitudinal study of 1058 adults (median age 75; 62\% women) who had cognitive function assessed and serum levels of 25-hydroxyvitaminD (25OHD) measured in 1997-99 and were followed for up to three additional cognitive function assessments over a 12year period.
\end{abstract}

\begin{abstract}
Results-Overall, 14\% ( $\mathrm{n}=145$ ) of participants had VitD insufficiency defined as $25 \mathrm{OHD}<30$ $\mathrm{ng} / \mathrm{ml}$. Adjusting for age, sex, education, and season, VitD insufficiency was associated with poorer baseline performance on the Mini-Mental Status Exam (MMSE) $(P=0.013)$, Trails Making Test B (Trails B) $(P=0.015)$, Category Fluency $(P=0.006)$ and Long Term Retrieval $(P=0.019)$; differences were equivalent to 5 years of age. For those with VitD insufficiency, the odds of mildly impaired performance at baseline were 38\% higher for MMSE $(P=0.08), 78 \%$ higher for Trails B $(P=0.017)$, and 2-fold higher for Category Fluency and Long Term Retrieval (both $P=0.001$ ). VitD insufficiency was not related to the rate of cognitive decline on any test or to the risk of developing impaired performance during follow-up.
\end{abstract}

Conclusion-In this population with little overt VitD deficiency, even moderately low VitD was associated with poorer performance on multiple domains of cognitive function. Low VitD did not predict 12-year cognitive decline. Clinical trials are essential to establish a causal link between VitD and cognitive well-being.

Correspondence: Gail A Laughlin, PhD, Department of Family Medicine and Public Health, University of California San Diego, 9500 Gilman Drive, MC0725, La Jolla, CA 92093, Phone: (858) 822-2416, Fax: (858) 534-4642, glaughlin@ucsd.edu. 


\section{Keywords}

cognitive aging; cognitive function; vitamin D; longitudinal study; epidemiology

\section{INTRODUCTION}

In addition to its well-established critical role in bone metabolism and emerging evidence of a role in cardiometabolic health, vitamin $\mathrm{D}(\mathrm{VitD})$ is now viewed as a neurosteroid with putative links to multiple brain functions including age-associated cognitive decline [1]. This thesis is supported by studies showing that both the VitD receptor and the enzyme that converts 25-hydroxyvitaminD [25OHD] to its active form 1,25-dihydroxyvitaminD are expressed in areas of the brain essential for cognition $[2,3]$. Understanding the contribution of VitD to cognitive function is critical given the aging of the population and estimates that as many as 1 billion individuals worldwide have inadequate VitD levels [4, 5].

Several epidemiological studies have examined the association of VitD concentrations with poorer cognitive function in older adults with mixed results depending on the population studied, its geographic location, the definition and degree of VitD deficiency, and the domain of cognitive function assessed [6,7]. Until recently, most studies have been cross-sectional and have examined only a few cognitive domains. Although it is not clear what level of VitD is sufficient to maintain cognitive health in aging, a recent review by the Institute of Medicine concluded that only the lowest levels of 25OHD $(<10 \mathrm{ng} / \mathrm{ml})$ were consistently associated with poorer cognitive function independent of other factors [8].

To our knowledge, the association between VitD and cognitive ability has not been evaluated in a relatively high functioning population of community-dwelling older adults residing in a temperate area with sunshine most days of the year and a low prevalence of VitD deficiency. This study examined the cross-sectional and prospective associations of circulating 25OHD levels with cognitive function and cognitive decline among older community-dwelling adults from the Rancho Bernardo Study (RBS), an observational cohort study of healthy aging. Longitudinal analyses included up to four cognitive function assessments over a 12-year period with multiple domains of cognitive function assessed and a wide range of potential confounding factors considered.

\section{METHODS}

\section{Study population}

Between 1972 and 1974, 82\% of all adults living in the southern California community of Rancho Bernardo were enrolled in a study of heart disease risk factors as part of the Lipid Research Clinics Prevalence Study [9]. Nearly all were middle to upper-middle class, $61 \%$ had attended at least some college, $33 \%$ had graduated. The present analysis included participants from the 1997-99 research clinic visit (baseline for this study) when blood samples for VitD measurement were collected and cognitive function was assessed. Cognitive function assessment was repeated at each of three subsequent study visits (19992003, 2003-06, 2007-09) providing a maximum of four assessments at approximate four- 
year intervals over a 12-year period. This study was approved by the Human Subjects Protection Program of the University of California San Diego. All participants provided written informed consent prior to participation.

Eligibility criteria for the present analysis included 1) age 50 years or older at baseline, 2) postmenopausal status for women (defined as at least one year since last menses), 3) availability of 25OHD measurements and 4) availability of cognitive function test results at the baseline visit. Of the 1098 participants who attended the 1997-99 visit, 13 were excluded for age less than 50 years, 18 for no $25 \mathrm{OHD}$ measurements, 7 for no cognitive function assessment and 2 women for premenopausal status. The final study sample consisted of 406 men and 652 women, for a total sample size of 1058 .

As part of the 1997-99 visit, information on medical history, medication use, physical activity, alcohol consumption and current smoking was obtained using standardized questionnaires. Current medication use was validated by examination of pills and prescriptions brought to the clinic for that purpose. Participants were asked to rate their overall health as compared to peers on a 5-point scale ranging from poor to excellent. Educational attainment was queried at entry into the cohort and classified as less than high school, high school graduate, some college, college graduate, or graduate school. Depressed mood was assessed using the Beck Depression Inventory (BDI) [10].

\section{Clinical measurements}

Height, weight, waist girth and hip girth were measured in the clinic with participants wearing light clothing and no shoes. Body mass index (BMI, $\mathrm{kg} / \mathrm{m}^{2}$ ) was used as an estimate of overall obesity; waist-hip ratio (WHR) as an estimate of central adiposity. Blood pressures were measured twice in seated resting subjects using the Hypertension Detection and Follow-Up Program protocol; the mean was used in analyses. Blood samples were obtained by venipuncture between 07:30 $\mathrm{AM}$ and 11:00 AM after a requested 12-hr fast; serum was separated and stored at $-70^{\circ} \mathrm{C}$ within 30 minutes of collection in tubes protected from sunlight. Plasma lipids and liver enzymes were determined by previously described methods [11]. Estimated glomerular filtration rate (eGFR) was calculated by the MDRD equation; participants with eGFR $<60$ were classified as having moderate chronic kidney disease [12]. Physical function was assessed using the Timed Up and Go (TUG) test, grip strength and observed gait impairment, as described previously [13].

\section{VitD determinations}

Serum $25[\mathrm{OH}] \mathrm{D})\left[25 \mathrm{OHD}_{2}+25 \mathrm{OHD}_{3}\right]$ was measured in the research laboratory of $\mathrm{M}$. Holick using VitD competitive binding protein recognition and chemiluminescence detection $[14,15]$. The intra- and inter-assay coefficients of variation for the 25OHD assay were 8 and $10 \%$, respectively [15]; the limit of detection was $5 \mathrm{ng} / \mathrm{mL}(13 \mathrm{nmol} / \mathrm{L})$, reference range $10-52 \mathrm{ng} / \mathrm{mL}(25-130 \mathrm{nmol} / \mathrm{L})$. Season was determined from the date of blood draw. We have previously shown that despite year-round sunshine, age-adjusted 25OHD levels are lower in this cohort in winter, and higher in fall [16]. The stability of VitD metabolites in frozen serum has been well described. Studies have demonstrated no detectable degradation of $25 \mathrm{OHD}$ in samples stored at $-20^{\circ} \mathrm{C}$ for over 10 years [17], and 
circulating $25 \mathrm{OHD}$ has been shown to be stable in stored serum samples, even after many freeze-thaw cycles [18].

\section{Cognitive function assessment}

At baseline and each follow-up visit, a standardized battery of cognitive function tests (CFT) was administered by an interviewer trained in neuropsychological testing, using consistent administration and scoring procedures across visits. Tests in the original task battery were selected in 1988 in conjunction with the University of California San Diego Alzheimer's Disease Research Center to incorporate those most likely to be sensitive to effects of aging and to assess a variety of cognitive domains. Three tests were administered at all visits. The Mini-Mental State Examination (MMSE) [19] assesses orientation, registration, attention, calculation, language and retrieval and is used as a test of global cognitive function. The Trail Making Test B (Trails B, from the Halstead-Reitan Neuropsychological Test Battery) tests visuomotor scanning and executive function [20]. Performance is rated by the time required to finish the test (maximum $300 \mathrm{sec}$ ); the higher the score, the poorer the test performance. The Animals Naming Category Fluency test assesses verbal semantic fluency and executive function [21], and is scored as the number of unique animals named in 60 seconds. A fourth test, the Buschke Selective Reminding test [22] was administered at the first three visits (due to time constraints, this test was not administered at the 2007-09 clinic visit). This test assesses long-term storage and retrieval of spoken words; lower scores indicate poorer performance. Long-term retrieval, the sum of all words retrieved from long term storage over the six reminder trials, was used as the memory outcome variable.

Mild test-specific impairment was defined as a score greater than 1.5 standard deviation (SD) below the sex-, age-, and education-adjusted mean for MMSE, Trails B and Category Fluency, based on normative data from the National Alzheimer's Coordinating Center Uniform Data Set (UDS) [23]. The Buschke-Fuld Selective Reminding test was not included in the UDS, thus impaired performance for Long Term Retrieval was defined as a score greater than 1.5 SD below the sex-, age- and education-adjusted mean for the study sample.

\section{Prevalent conditions}

Prevalent CVD was defined as self-report of physician-diagnosed myocardial infarction, coronary artery revascularization, congestive heart failure, stroke or transient ischemic attack, carotid surgery, peripheral artery surgery, or physician-diagnosed intermittent claudication. Diabetes was defined by self-reported physician diagnosis, fasting plasma glucose $\searrow 6.99 \mathrm{mmol} / \mathrm{L}$, or use of diabetes medications. Hypertension was defined as blood pressure $\geq 140 / 90 \mathrm{~mm} \mathrm{Hg}$ or use of antihypertensive medication. The metabolic syndrome was defined according to NCEP ATP III criteria [24]. Stroke risk was assessed using the Framingham Risk Score criteria [25, 26], with the exception of left ventricular hypertrophy, which was not available. Comorbidities recorded included atrial fibrillation, angina, congestive heart failure, stroke, transient ischemic attack, myocardial infarction, angioplasty, arthritis, diabetes, asthma, cancer (non-skin), emphysema, kidney disease, thyroid disease, ulcer, osteoporosis, hip fracture, Parkinson's disease, and hypertension. 


\section{Statistical Analyses}

Based on prevailing hypothesized cutpoints [27-29], VitD insufficiency was defined as $25 \mathrm{OHD}<30 \mathrm{ng} / \mathrm{ml}$. Participant characteristics at baseline were compared for those with and without VitD insufficiency using general linear models (GLM) adjusting for age and sex. The relation of VitD insufficiency to baseline CFT performance and change in performance over time was examined using linear mixed effects regression models. This statistical approach handles missing data and inconsistent measurement intervals within and across participants and accounts for within-subject correlation between repeated measures. Separate models were created for each CFT. Models included fixed effect terms, which model the mean trajectory of subjects as a function of the covariates: baseline age, sex, education, season of sample blood draw, and time (years since baseline). Models also included random effect coefficients, which allow individual subject baseline levels (intercept) and rates of decline (slope) to vary randomly about the mean trajectory described by the fixed effect terms. Interaction terms of all covariates with time were included to test whether the influence changed over time. Quadratic (squared) terms for time were included in all models because of the long follow-up period (up to 12 years). Participants may improve on CFT with retest, due to familiarity with the tests. Retest effects were evaluated by including a retest term in the model. Based on prior findings, we assumed that the largest effect of retest would occur between the first and second assessments [30]. Thus, the retest variable was defined as zero on the participant's first cognitive assessment and as one on all subsequent assessments [31].

The proportion of participants with poor cognitive performance was compared for those with and without VitD insufficiency for each CFT using GLM. Associations between VitD insufficiency and poor cognitive performance at baseline were tested by logistic regressions. Incident cognitive impairment for each CFT was determined among those without cognitive impairment at baseline who returned for at least one, and up to three, additional cognitive function assessments. Time to event for incident impairment cases for each CFT was defined as the time between baseline and the first occurrence of CFT scores below the respective 1.5 SD thresholds. Non-cases were censored at the time of their last cognitive assessment. Associations between VitD insufficiency and incident cognitive impairment were assessed using Cox proportional hazards regressions.

The same general covariate modeling approach was used for all analyses, i.e. mixed effects linear regressions, logistic regressions and Cox proportional hazards regression. Base models were adjusted for age, sex, education, and season of blood draw. A second model tested confounding by body size and lifestyle by adding adjustment for BMI, WHR, smoking, exercise and alcohol use. A third model added adjustment for markers of health status related to VitD insufficiency in this cohort at $P<0.20$ (heart rate, kidney function [eGFR], liver function [AST, ALT], self-assessed health, diabetes, metabolic syndrome, physical function [timed up and go, impaired gait]), as well as depression scores. All models for incident cognitive impairment were additionally adjusted for baseline CFT score.

Because thresholds for cognitive impairment were adjusted for sex, age and education, these covariates were not included in models for cognitive impairment analyses. Interactions of VitD insufficiency by sex, age and BMI were tested for base regression models as 
appropriate; none were significant. To address bias that may result from including individuals with poor health, we repeated all analyses excluding 93 participants who rated their overall health as "fair" or "poor" compared to peers of the same age; thus restricting the sample to those reporting "good", "very good" or "excellent" health at baseline ( $\mathrm{N}=965)$. Sensitivity analyses tested the influence of excluding 24 participants with VitD $<20 \mathrm{ng} / \mathrm{ml}$, three of these were $<10 \mathrm{ng} / \mathrm{ml}$.

Exact $P$-values for two-sided tests are shown; $P<0.05$ was considered to be statistically significant for all analyses including interaction terms. Data were analyzed using SAS (v9.4, SAS Institute, Inc., Cary, NC) or SPSS (v18.0; SPSS Inc., Chicago, IL).

\section{RESULTS}

\section{Participant characteristics}

Participant characteristics at baseline are presented in Table 1 . The mean age of the 1058 participants was 75 years (range 50 to 97$) ; 62 \%(n=652)$ were women of whom $39 \%$ reported current use of oral estrogens. Overall, $41 \%$ were college graduates, $4 \%$ were current smokers and $72 \%$ reported exercising 3 or more times per week. Median 25OHD levels were $40 \mathrm{ng} / \mathrm{ml} ; 145$ (13.5\%) participants had levels $<30 \mathrm{ng} / \mathrm{ml}, 24$ (2.3\%) had levels $<20 \mathrm{ng} / \mathrm{ml}$ and three had levels $<10 \mathrm{ng} / \mathrm{ml}$.

Age- and sex-adjusted characteristics by VitD insufficiency $(25 \mathrm{OHD}<30 \mathrm{ng} / \mathrm{ml}$ ) versus higher VitD levels are shown in Table 1. Individuals with VitD insufficiency were older, more likely to be female, more likely to report fair or poor health, and had higher BMI, WHR and resting heart rate compared to those with adequate VitD levels (all $P<0.01$ ). They were also more likely to have diabetes $(P=0.03)$ and metabolic syndrome ( $P=0.01)$, less likely to exercise regularly $(P<0.01)$, had slower times on the Timed Up and Go mobility test $(P<0.001)$, fewer hours of outdoor activity weekly ( 3.2 vs $5.2 \mathrm{hrs}, P<0.001)$, and drank alcohol less frequently $(P<0.01)$. Only $4 \%$ of those with VitD insufficiency reported use of VitD supplements compared to $24 \%$ of those with adequate VitD levels $(P<0.001)$.

\section{VitD Insufficiency and Cognitive Function}

Cognitive function assessments were available at up to four research clinic visits, including the baseline visit. Overall, $43 \%$ of participants completed all four assessments, $17 \%$ completed three, $22 \%$ completed two, and $18 \%$ completed one. The VitD insufficiency group had an average of 3.0 assessments compared to 3.3 for those with adequate VitD levels and a mean follow-up period of 6.4 years (maximum 11.9) versus 7.3 years (maximum 12.0) ( $P<0.01$ for both). The mean age at last assessment was 80.1 years for those with VitD insufficiency and 83.0 years for those with adequate VitD $(P=0.001)$.

Figure 1 shows modeled trajectories of cognitive function over time as a function of VitD insufficiency for each CFT. These plots are based on all model coefficients using VitD group-specific mean values for covariates. Parameter estimates for the influence of VitD insufficiency are presented in Table 2. Values for the main effect of low VitD indicate the influence of VitD insufficiency on scores at baseline (intercept); values for low VitD by time 
show the influence of baseline VitD insufficiency on change in cognitive function score during follow-up (slope).

VitD insufficiency was associated with significantly poorer scores at baseline on MMSE, Trails B, Category Fluency, and Long Term Retrieval adjusting for age, sex, education and season of blood draw (Model 1). These differences remained significant after adding adjustment for body size and lifestyle (Model 2) and were marginally significant $(P<0.06)$ after additional adjustment for markers of health status and depression (Model 3) for all tests except Long Term Retrieval. For Model 1, the magnitude of the influence of VitD insufficiency on baseline CFT scores was comparable to that of a 5-year increase in age, which was -0.4 points for MMSE, +17.6 points for Trails B, -1.0 point for Category Fluency, and -2.2 points for Long Term Retrieval (all $P<0.001$ ). VitD insufficiency was not significantly related to the rate of change in performance for any CFT during the 12-year follow-up; however, the baseline decrement in performance for those with VitD insufficiency was sustained throughout the follow-up for Trails B and remained lower for Long Term Retrieval

Compared to the Healthy Subset analyzed in the sensitivity analysis (Table 2, lower panel), the 93 excluded individuals with poorer self-assessed health were more likely to have diabetes, metabolic syndrome, hypertension, and prevalent CVD (all $\mathrm{P}<0.001$, data not shown). In addition, they had more comorbidities, took more medications, and had more doctors visits and time in the hospital in the past year (all $\mathrm{P}<0.01$, data not shown). Thus, this self-report variable encompasses a number of explicit measures of health status. Exclusion of those with poorer self-assessed health increased the significance of baseline differences for the fully-adjusted model for all CFTs.

\section{VitD Insufficiency and Impaired Cognitive Function}

As shown in Figure 2, the proportion of participants scoring in the mildly impaired range (1.5 SD below age, sex and education adjusted norms) at baseline was significantly higher in the VitD insufficient group than the VitD sufficient group for each CFT. For those with VitD insufficiency, the odds of poor cognitive function were $38 \%$ higher for MMSE $(P=0.08)$, $78 \%$ higher for Trails B $(P=0.017)$, and 2-fold higher ( $P=0.001)$ for Category Fluency and Long Term Retrieval adjusting for the season of blood draw (Table 3). Associations with poor cognitive function remained significant for Trails B, Category Fluency and Long Term Retrieval after additional adjustment for body size and lifestyle (Model 2), but were only significant for Category Fluency and Long Term Retrieval after adjusting for multiple markers of health status (Model 3). In analyses excluding 93 participants who reported poorer health, associations of VitD insufficiency with cognitive impairment were moderately stronger for MMSE, Trails B and Long Term Retrieval.

Next, we summed the number of CFT scores in the impaired range for each individual. The prevalence of VitD insufficiency increased continuously as the total number of CFT scores in the impaired range increased for both the full sample and the healthy subset (Figure 3). Overall, $46 \%$ of those with VitD insufficiency had at least one impaired score and $21 \%$ had two or more, as compared to $35 \%$ and $11 \%$, respectively, for those with adequate VitD (both $P<0.001)$. 
The association of VitD insufficiency with incident cognitive impairment was examined in those without cognitive impairment at baseline (Table 4). VitD insufficiency was not related to the development of impairment for any CFT during the 12-year follow-up period for the full sample or for the healthy subset.

\section{Sensitivity Analyses}

Longitudinal analyses were repeated using VitD as a continuous variable with similar findings of lower baseline CFT scores in those with VitD insufficiency and no significant associations with the rate of change (Supplementary Table 1). To further define the influence of VitD insufficiency, exclusive of VitD deficiency, analyses were repeated excluding the 24 participants with $25 \mathrm{OHD}<20 \mathrm{ng} / \mathrm{ml}$. As shown in Supplementary Table 2, baseline differences for those with VitD insufficiency, now defined as $20 \leq 25 \mathrm{OHD}<30 \mathrm{ng} / \mathrm{ml}$, were somewhat stronger for all CFTs in the fully-adjusted model (Model 3) for both the full sample and the healthy subset. Cross-sectional results for scoring in the mildly impaired range were essentially unchanged (Supplementary Table 3 ). VitD insufficiency continued to be unrelated to either the rate of change in CFT performance during follow-up (Supplementary Table 2) or the development of impairment (data not shown) for any CFT.

\section{DISCUSSION}

Overt VitD deficiency was rare in this population of older adults living in a southern California community with sunshine on most days of the year. However, VitD insufficiency $(25 \mathrm{OHD}<30 \mathrm{ng} / \mathrm{ml}$ ) was present in $14 \%$ of the study sample and was associated with poorer performance on four cognitive function tests assessing global cognitive function, executive function, verbal semantic fluency and verbal episodic memory. These findings were independent of a large number of confounders and covariates including multiple indicators of health status and physical function. Those with VitD insufficiency were also more likely to be mildly impaired, or worse, on these tests, based on normative scores for similar populations. In contrast to these cross-sectional findings, VitD insufficiency was not associated with the rate of decline in cognitive performance over the following 12-years or with the development of impaired function for any of the CFTs administered.

These results add to prior studies showing a cross-sectional association of very low circulating 25OHD levels and poorer cognitive function in older adults, and extend these findings by demonstrating a negative influence of even insufficient serum VitD in a relatively high-functioning population with year round sunshine and little VitD deficiency. Systematic reviews and meta-analyses have reported a consistent cross-sectional association of hypovitaminosis D with global cognitive impairment in older adults [6,7], as well as a strong link with executive dysfunction [32]; results for other cognitive domains are less certain [7, 32]. Importantly, most prior studies found associations only for very low levels of VitD; $<10 \mathrm{ng} / \mathrm{ml}$ in most cases, $<20 \mathrm{ng} / \mathrm{ml}$ in others. To our knowledge, our study is unique in having examined and found a consistent negative association of VitD $<30 \mathrm{ng} / \mathrm{ml}$ across multiple cognitive domains, even after excluding individuals with poorer health and the few $(n=24)$ with VitD <20. We also found that the prevalence of VitD insufficiency correlated strongly with the number of impaired cognitive domains. 
An international task force recently concluded that in addition to cross-sectional associations, very low levels of VitD are also a reliable risk factor for predicting future cognitive decline and incident dementia in the elderly [28]. We failed to find a prospective association of VitD insufficiency with the rate of decline over a 12-year follow period or with development of impairment for any of the four cognitive tests assessed. Because most literature suggests an increased risk of cognitive decline in individuals with VitD levels below $10 \mathrm{ng} / \mathrm{ml}$ [6-8], our results may mean that only larger disruptions in 25OHD contribute to the rate of cognitive decline in older adults. However, not all prospective studies have found significant associations, even for VitD levels below $10 \mathrm{ng} / \mathrm{ml}$. In MrOS (Osteoporotic Fractures in Men) and SOF (Study of Osteoporotic Fractures), Slinin and colleagues found that $25 \mathrm{OHD}<10 \mathrm{ng} / \mathrm{ml}$ was associated with 4 to 5 year decline in global cognitive function for older women [33], but not older men [34], and VitD deficiency did not relate to changes in executive function in either sex. In the ARIC (Atherosclerosis Risk in Communities) Study [35], lower levels of 25OHD were not significantly associated with lower cognitive test scores at baseline or with greater decline in cognitive test scores over 3 and 11-year follow-ups. Prospective studies of memory have also had variable results; low VitD was associated with visual memory decline in the Cardiovascular Health Study (CHS), but not with verbal memory change in the Longitudinal Aging Study Amsterdam (LASA) [36]. It is notable that our study, like most other prospective studies of VitD and cognitive function, is based on a single baseline measurement of VitD. The follow-up took place during a period when clinical screening and sales of VitD supplements increased substantially [37, 38], thus the VitD status of individual participants may have changed, confounding our ability to identify long-term influences. Prospective studies with assessment of VitD at multiple time points may resolve these divergent results, although observational studies have other inherent drawbacks.

Studies of VitD and cognitive function are limited by the possibility of reverse causality, and this is especially true for cross-sectional studies and those with short-term follow-up. VitD is derived from the diet and sunlight and is sequentially activated in the liver and kidneys [39]. Low VitD in the elderly may be due to multiple factors including reduced sun exposure, inadequate food intake, sequestration in fat, and altered metabolism due to impaired hepatic and renal function [40], which themselves can affect cognitive function [41]. In the present study, we were able to adjust for a large number of health and mobility measures, including direct measures of physical function. Only a few VitD associations lost significance and effect sizes were not greatly attenuated, allowing us to be more confident that the observed associations were not due to reverse causation. In addition, excluding individuals with poorer health tended to strengthen, rather than eliminate, associations.

Concerns about causality are somewhat mitigated by the abundance of biological evidence supporting neuroprotective effects of VitD $[42,43]$. VitD receptors are plentiful in the hippocampi and cortex, and the enzymes critical for VitD activity are expressed in neurons and glial cells [2,3]. VitD may help to prevent neurodegeneration through multiple pathways including antioxidative mechanisms, regulation of calcium homeostasis, immunomodulation, and detoxification [43, 44]. In addition, VitD stimulates neurogenesis and regulates the synthesis of neurotrophic factors important for cell differentiation and survival [43], and is linked with amyloid phagocytosis and clearance [45]. It is also possible 
that VitD protects brain function via pathways related to the development and progression of peripheral and cerebral vascular diseases [46].

While the clinical implications of our findings cannot be stated with certainty, the effect sizes associated with VitD insufficiency appear meaningful. For example, the difference in baseline cognitive performance for those with lower VitD was equivalent to a 5-year increase in age for each of the four tests. In addition, the difference in Trails B scores associated with VitD insufficiency in our study (main effect size $=11.7$ ) was similar to a mean difference of 12.5 for Trails B in individuals with and without VitD deficiency derived from combining four other observational studies [32]. Finally, the possibility of even mildly impaired performance on tests of verbal fluency and verbal episodic memory was 2-fold higher in those with VitD insufficiency, even after accounting for a large number of potential confounders. This level of risk is strikingly similar to that identified for VitD deficiency and coincident cognitive impairment in a recent meta-analysis [7]. Given the rapidly growing elderly population, identification of factors associated with even moderate differences in cognitive function may have a large public health impact. VitD insufficiency may be one such factor.

Although our study is in agreement with prior studies showing a negative association of low VitD with cognitive function, only long-term, randomized placebo-controlled trials can determine with certainty whether maintenance of 25OHD above a specific threshold will slow cognitive aging, and where that threshold lies. Thus far, two prior randomized controlled trials, one in younger and one in older participants $[47,48]$ and a post-hoc analysis of the Women's Health Initiative (WHI) Calcium and Vitamin D Trial [49] did not find a beneficial effect of VitD supplementation (400-5000 IU/day) on cognitive function. Several large (>10,000 participants) and moderate (2000-10,000 participants) scale randomized trials of VitD supplementation are underway in the United States, Europe and Australia [50]. Although none have cognitive function as a primary endpoint, many include cognitive testing as a secondary or ancillary outcome and should provide vital information about the cognitive benefits of VitD supplementation.

Our study has a number of important strengths including the large sample size, inclusion of both sexes, assessment of multiple domains of cognitive function up to four times over a 12year follow-up period, and availability of a large number of potential confounders and covariates including multiple comorbidities and markers of health status. Some possible limitations should also be considered. The RBS participants are almost all Caucasian, middle- to upper-middle class, and relatively healthy. This may limit generalizability, but is a strength to the extent that it greatly diminishes confounding due to race/ethnicity, socioeconomic status and access to health care. More importantly, RBS participants live in a temperate climate with year-round sun exposure, and thus have little 25OHD deficiency. These results may not be generalizable to populations with more VitD deficiency, but do yield novel information about potential risks conferred by lesser degrees of VitD insufficiency and its importance in warmer, sunnier climates. It is also possible that the higher VitD levels in our population are due to differences in assay techniques. Competitive binding protein assay may produce higher $25 \mathrm{OHD}$ results compared to radioimmunoassay and high-performance liquid chromatography [51, 52], thus levels in RBS may not be 
directly comparable to studies using different assays. Nevertheless, routine assays accurately rank individuals across the range of 25OHD levels [52], thus the internal validity of our study is not compromised, and results were similar in analyses using VitD as a continuous variable. In addition, higher assay measurements would underestimate the number of individuals with VitD insufficiency, therefore biasing findings to the null. Values for 25OHD were measured on a single blood specimen and VitD is known to have seasonal variation $[16,53]$. This may have weakened the cognitive function associations, but is unlikely to have caused them. In addition, our models adjusted for season of blood draw, thus associations were independent of seasonality. Another limitation, common to all studies of the elderly, is survival bias; persons with greater VitD deficiency may have died sooner or have been too ill to return to the follow-up visits, potentially attenuating any true association. Although statistically different, the mean number of repeat assessments and age at last cognitive assessment did not differ markedly for the VitD sufficient and insufficient groups (3.3 vs 3.0 assessments and age 83.0 vs 80.1 years, respectively), suggesting survival bias did not play a major role in our findings.

In conclusion, this study provides new evidence that VitD levels below $30 \mathrm{ng} / \mathrm{ml}$, widely considered to be the threshold for VitD insufficiency, are associated with clinically meaningful reductions in cognitive function in community-dwelling older adults living in a temperate climate with year round sunshine. These associations applied to multiple cognitive domains, as well as the risk of cognitive impairment, suggesting that a sufficient level of VitD may be necessary to maintain optimal cognitive function with aging. Baseline VitD insufficiency was not related to the rate of cognitive decline over the next decade and did not predict future cognitive impairment. Carefully designed randomized, controlled trials of VitD supplementation are essential to establish the causal nature of these findings and to determine whether maintaining $25 \mathrm{OHD}$ above a critical threshold will contribute to the preservation of cognitive ability with aging, especially in populations with a higher prevalence of VitD deficiency.

\section{Supplementary Material}

Refer to Web version on PubMed Central for supplementary material.

\section{Acknowledgments}

This study was supported by a grant from the National Institute on Alcohol Abuse and Alcoholism (R01AA021187). The Rancho Bernardo Study was funded by research grants AG028507 and AG007181 from the National Institute on Aging and grant DK31801 from the National Institute of Diabetes and Digestive and Kidney Diseases.

\section{References}

1. Stewart A, Wong K, Cachat J, Elegante M, Gilder T, Mohnot S, Wu N, Minasyan A, Tuohimaa P, Kalueff AV. Neurosteroid vitamin D system as a nontraditional drug target in neuropsychopharmacology. Behav Pharmacol. 2010; 21:420-426. [PubMed: 20571365]

2. Eyles DW, Liu PY, Josh P, Cui X. Intracellular distribution of the vitamin D receptor in the brain: comparison with classic target tissues and redistribution with development. Neuroscience. 2014; 268:1-9. [PubMed: 24607320] 
3. Eyles DW, Smith S, Kinobe R, Hewison M, McGrath JJ. Distribution of the vitamin D receptor and 1 alpha-hydroxylase in human brain. J Chem Neuroanat. 2005; 29:21-30. [PubMed: 15589699]

4. Annweiler C, Souberbielle JC, Schott AM, de Decker L, Berrut G, Beauchet O. Vitamin D in the elderly: 5 points to remember. Geriatr Psychol Neuropsychiatr Vieil. 2011; 9:259-267. [PubMed: 21896429]

5. Holick MF. Vitamin D deficiency. N Engl J Med. 2007; 357:266-281. [PubMed: 17634462]

6. Annweiler C, Allali G, Allain P, Bridenbaugh S, Schott AM, Kressig RW, Beauchet O. Vitamin D and cognitive performance in adults: a systematic review. Eur J Neurol. 2009; 16:1083-1089. [PubMed: 19659751]

7. Etgen T, Sander D, Bickel H, Sander K, Forstl H. Vitamin D deficiency, cognitive impairment and dementia: a systematic review and meta-analysis. Dement Geriatr Cogn Disord. 2012; 33:297-305. [PubMed: 22759681]

8. Medicine) IIo. Cognitive aging: Progress in understanding and opportunities for action. The National Academies Press; Washington. D.C: 2015.

9. Criqui MH, Barrett-Connor E, Austin M. Differences between respondents and non-respondents in a population-based cardiovascular disease study. Am J Epidemiol. 1978; 108:367-372. [PubMed: 727205]

10. Beck AT, Ward CH, Mendelson M, Mock J, Erbaugh J. An inventory for measuring depression. Arch Gen Psychiatry. 1961; 4:561-571. [PubMed: 13688369]

11. Laughlin GA, Barrett-Connor E, Bergstrom J. Low serum testosterone and mortality in older men. J Clin Endocrinol Metab. 2008; 93:68-75. [PubMed: 17911176]

12. K/DOQI clinical practice guidelines for chronic kidney disease: evaluation, classification, and stratification. Am J Kidney Dis. 2002; 39:S1-266. [PubMed: 11904577]

13. Dam TT, von Muhlen D, Barrett-Connor EL. Sex-specific association of serum vitamin D levels with physical function in older adults. Osteoporos Int. 2009; 20:751-760. [PubMed: 18802657]

14. Chen TC, Turner AK, Holick MF. A method for the determination of the circulating concentration of 1,25-dihydroxyvitamin D. J Nutr Biochem. 1990; 1:320-327. [PubMed: 15539222]

15. Chen TC, Turner AK, Holick MF. Methods for the determination of the circulating concentration of 25-hydroxyvitamin D. J Nutr Biochem. 1990; 1:315-319. [PubMed: 15539221]

16. von Muhlen DG, Greendale GA, Garland CF, Wan L, Barrett-Connor E. Vitamin D, parathyroid hormone levels and bone mineral density in community-dwelling older women: the Rancho Bernardo Study. Osteoporos Int. 2005; 16:1721-1726. [PubMed: 15928802]

17. Hollis BW. Measuring 25-hydroxyvitamin D in a clinical environment: challenges and needs. Am J Clin Nutr. 2008; 88:507s-510s. [PubMed: 18689391]

18. Antoniucci DM, Black DM, Sellmeyer DE. Serum 25-hydroxyvitamin D is unaffected by multiple freeze-thaw cycles. Clin Chem. 2005; 51:258-261. [PubMed: 15613728]

19. Tombaugh TN, McIntyre NJ. The mini-mental state examination: a comprehensive review. J Am Geriatr Soc. 1992; 40:922-935. [PubMed: 1512391]

20. Reitan R. Validity of the trail-making test as an indicator of organic brain disease. Percept Mot Skills. 1958; 8:271-276.

21. Borkowski J, Benton AL, Spreen O. Word fluency and brain damage. Neuropsychologia. 1967; 5:135-140.

22. Gregg EW, Yaffe K, Cauley JA, Rolka DB, Blackwell TL, Narayan KM, Cummings SR. Is diabetes associated with cognitive impairment and cognitive decline among older women? Study of Osteoporotic Fractures Research Group. Arch Intern Med. 2000; 160:174-180. [PubMed: 10647755]

23. Shirk SD, Mitchell MB, Shaughnessy LW, Sherman JC, Locascio JJ, Weintraub S, Atri A. A webbased normative calculator for the uniform data set (UDS) neuropsychological test battery. Alzheimers Res Ther. 2011; 3:32. [PubMed: 22078663]

24. Executive Summary of The Third Report of The National Cholesterol Education Program (NCEP) Expert Panel on Detection, Evaluation, And Treatment of High Blood Cholesterol In Adults (Adult Treatment Panel III). JAMA. 2001; 285:2486-2497. [PubMed: 11368702] 
25. D’Agostino RB, Wolf PA, Belanger AJ, Kannel WB. Stroke risk profile: adjustment for antihypertensive medication. The Framingham Study. Stroke. 1994; 25:40-43. [PubMed: 8266381]

26. Wolf PA, D’Agostino RB, Belanger AJ, Kannel WB. Probability of stroke: a risk profile from the Framingham Study. Stroke. 1991; 22:312-318. [PubMed: 2003301]

27. Annweiler C, Beauchet O. Vitamin d in older adults: the need to specify standard values with respect to cognition. Front Aging Neurosci. 2014; 6:72. [PubMed: 24782767]

28. Annweiler C, Dursun E, Feron F, Gezen-Ak D, Kalueff AV, Littlejohns T, Llewellyn DJ, Millet P, Scott T, Tucker KL, Yilmazer S, Beauchet O. 'Vitamin D and cognition in older adults': updated international recommendations. J Intern Med. 2015; 277:45-57. [PubMed: 24995480]

29. Holick MF, Binkley NC, Bischoff-Ferrari HA, Gordon CM, Hanley DA, Heaney RP, Murad MH, Weaver CM. Evaluation, treatment, and prevention of vitamin D deficiency: an Endocrine Society clinical practice guideline. J Clin Endocrinol Metab. 2011; 96:1911-1930. [PubMed: 21646368]

30. Bartels C, Wegrzyn M, Wiedl A, Ackermann V, Ehrenreich H. Practice effects in healthy adults: a longitudinal study on frequent repetitive cognitive testing. BMC Neurosci. 2010; 11:118. [PubMed: 20846444]

31. Ferrer E, Salthouse TA, Stewart WF, Schwartz BS. Modeling age and retest processes in longitudinal studies of cognitive abilities. Psychol Aging. 2004; 19:243-259. [PubMed: 15222818]

32. Annweiler C, Montero-Odasso M, Llewellyn DJ, Richard-Devantoy S, Duque G, Beauchet O. Meta-analysis of memory and executive dysfunctions in relation to vitamin D. J Alzheimers Dis. 2013; 37:147-171. [PubMed: 23948884]

33. Slinin Y, Paudel M, Taylor BC, Ishani A, Rossom R, Yaffe K, Blackwell T, Lui LY, Hochberg M, Ensrud KE. Association between serum 25(OH) vitamin D and the risk of cognitive decline in older women. J Gerontol A Biol Sci Med Sci. 2012; 67:1092-1098. [PubMed: 22454371]

34. Slinin Y, Paudel ML, Taylor BC, Fink HA, Ishani A, Canales MT, Yaffe K, Barrett-Connor E, Orwoll ES, Shikany JM, Leblanc ES, Cauley JA, Ensrud KE. 25-Hydroxyvitamin D levels and cognitive performance and decline in elderly men. Neurology. 2010; 74:33-41. [PubMed: 19940271]

35. Schneider AL, Lutsey PL, Alonso A, Gottesman RF, Sharrett AR, Carson KA, Gross M, Post WS, Knopman DS, Mosley TH, Michos ED. Vitamin D and cognitive function and dementia risk in a biracial cohort: the ARIC Brain MRI Study. Eur J Neurol. 2014; 21:1211-1218. e1269-1270. [PubMed: 24846449]

36. Kuzma E, Soni M, Littlejohns TJ, Ranson JM, van Schoor NM, Deeg DJ, Comijs H, Chaves PH, Kestenbaum BR, Kuller LH, Lopez OL, Becker JT, Langa KM, Henley WE, Lang IA, Ukoumunne OC, Llewellyn DJ. Vitamin D and Memory Decline: Two Population-Based Prospective Studies. J Alzheimers Dis. 2016

37. LeFevre ML. Screening for vitamin D deficiency in adults: U.S. Preventive Services Task Force recommendation statement. Ann Intern Med. 2015; 162:133-140. [PubMed: 25419853]

38. Gahche J, Bailey R, Burt V, Hughes J, Yetley E, Dwyer J, Picciano MF, McDowell M, Sempos C. Dietary supplement use among U.S. adults has increased since NHANES III (1988-1994). NCHS Data Brief. 2011:1-8.

39. Gallagher JC. Vitamin D and aging. Endocrinol Metab Clin North Am. 2013; 42:319-332. [PubMed: 23702404]

40. Dusso AS, Brown AJ, Slatopolsky E. Vitamin D. Am J Physiol Renal Physiol. 2005; 289:F8-28. [PubMed: 15951480]

41. Shatenstein B, Barberger-Gateau P, Mecocci P. Prevention of Age-Related Cognitive Decline: Which Strategies, When, and for Whom? J Alzheimers Dis. 2015; 48:35-53. [PubMed: 26401926]

42. Buell JS, Dawson-Hughes B. Vitamin D and neurocognitive dysfunction: preventing “D”ecline? Mol Aspects Med. 2008; 29:415-422. [PubMed: 18579197]

43. McCann JC, Ames BN. Is there convincing biological or behavioral evidence linking vitamin D deficiency to brain dysfunction? Faseb j. 2008; 22:982-1001. [PubMed: 18056830]

44. Garcion E, Wion-Barbot N, Montero-Menei CN, Berger F, Wion D. New clues about vitamin D functions in the nervous system. Trends Endocrinol Metab. 2002; 13:100-105. [PubMed: 11893522] 
45. Masoumi A, Goldenson B, Ghirmai S, Avagyan H, Zaghi J, Abel K, Zheng X, Espinosa-Jeffrey A, Mahanian M, Liu PT, Hewison M, Mizwickie M, Cashman J, Fiala M. 1alpha,25-

dihydroxyvitamin D3 interacts with curcuminoids to stimulate amyloid-beta clearance by macrophages of Alzheimer's disease patients. J Alzheimers Dis. 2009; 17:703-717. [PubMed: 19433889]

46. McGreevy C, Williams D. New insights about vitamin D and cardiovascular disease: a narrative review. Ann Intern Med. 2011; 155:820-826. [PubMed: 22184689]

47. Stein MS, Scherer SC, Ladd KS, Harrison LC. A randomized controlled trial of high-dose vitamin D2 followed by intranasal insulin in Alzheimer's disease. J Alzheimers Dis. 2011; 26:477-484. [PubMed: 21694461]

48. Dean AJ, Bellgrove MA, Hall T, Phan WM, Eyles DW, Kvaskoff D, McGrath JJ. Effects of vitamin D supplementation on cognitive and emotional functioning in young adults--a randomised controlled trial. PLoS One. 2011; 6:e25966. [PubMed: 22073146]

49. Rossom RC, Espeland MA, Manson JE, Dysken MW, Johnson KC, Lane DS, LeBlanc ES, Lederle FA, Masaki KH, Margolis KL. Calcium and vitamin D supplementation and cognitive impairment in the women's health initiative. J Am Geriatr Soc. 2012; 60:2197-2205. [PubMed: 23176129]

50. Manson JE, Bassuk SS. Vitamin D research and clinical practice: at a crossroads. Jama. 2015; 313:1311-1312. [PubMed: 25695911]

51. Binkley N, Krueger D, Cowgill CS, Plum L, Lake E, Hansen KE, DeLuca HF, Drezner MK. Assay variation confounds the diagnosis of hypovitaminosis D: a call for standardization. J Clin Endocrinol Metab. 2004; 89:3152-3157. [PubMed: 15240586]

52. Lips P, Chapuy MC, Dawson-Hughes B, Pols HA, Holick MF. An international comparison of serum 25-hydroxyvitamin D measurements. Osteoporos Int. 1999; 9:394-397. [PubMed: 10550457]

53. Stamp TC, Round JM. Seasonal changes in human plasma levels of 25-hydroxyvitamin D. Nature. 1974; 247:563-565. [PubMed: 4818557] 

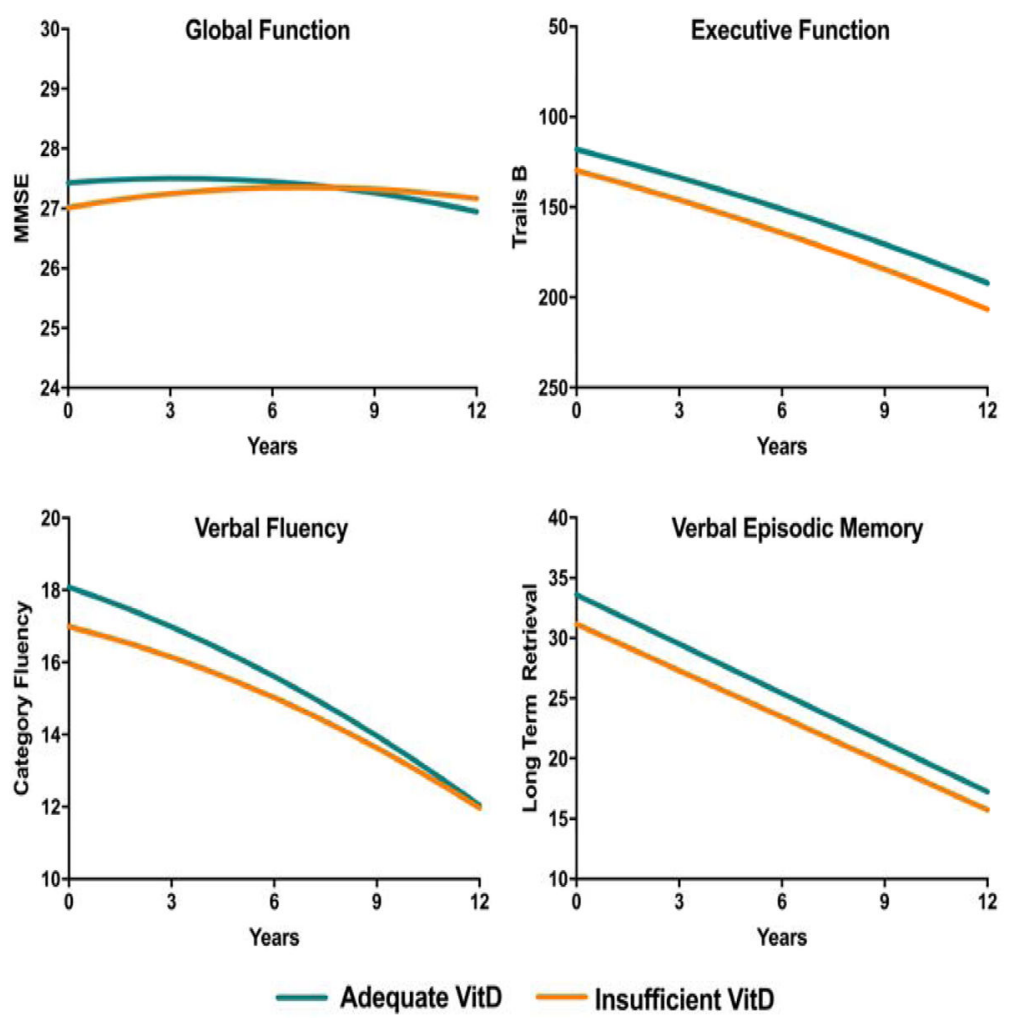

Figure 1.

Modeled trajectories of cognitive function test performance over time as a function of VitD status. Plots are based on all model coefficients using VitD group-specific mean values for covariates: age, sex, season of sample draw, education and practice effect. Models for VitD insufficient participants are shown in orange, VitD sufficiency in teal. The axis for Trails B is reversed so that for all tests, downward sloping lines show decreasing performance. 


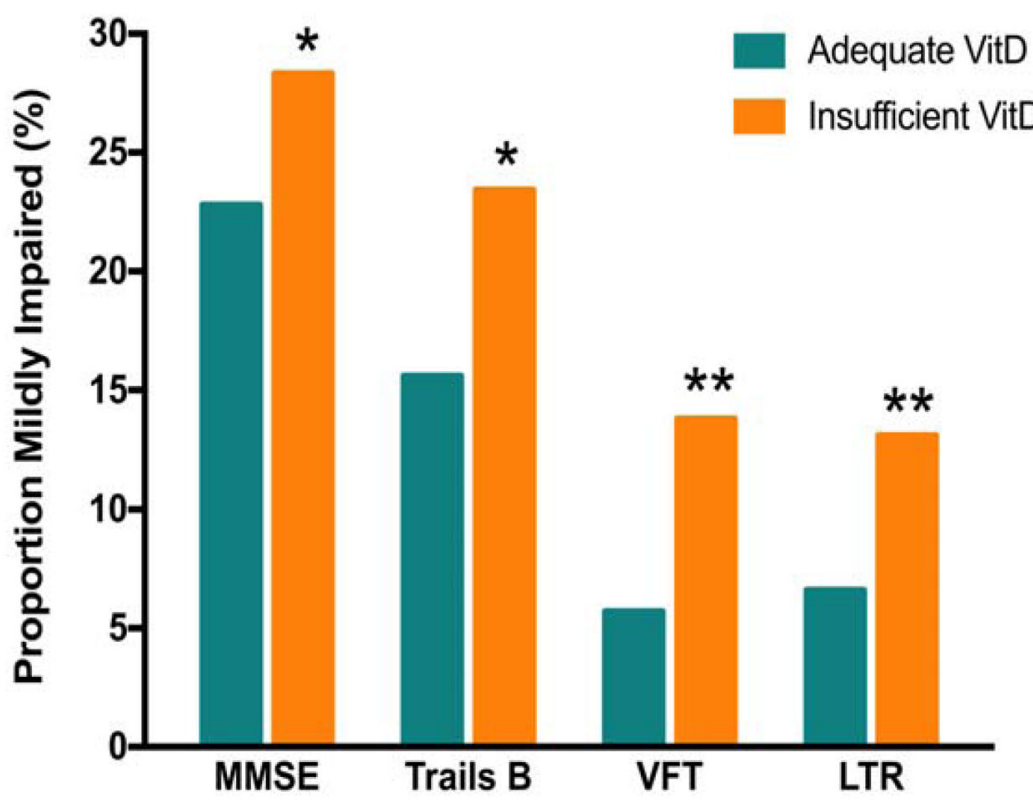

Figure 2.

Prevalence of mildly impaired performance for each CFT by VitD insufficiency (orange) and sufficiency (teal). * indicates $P<0.05, * *$ indicates $P<0.01$ comparing adequate to insufficient VitD 


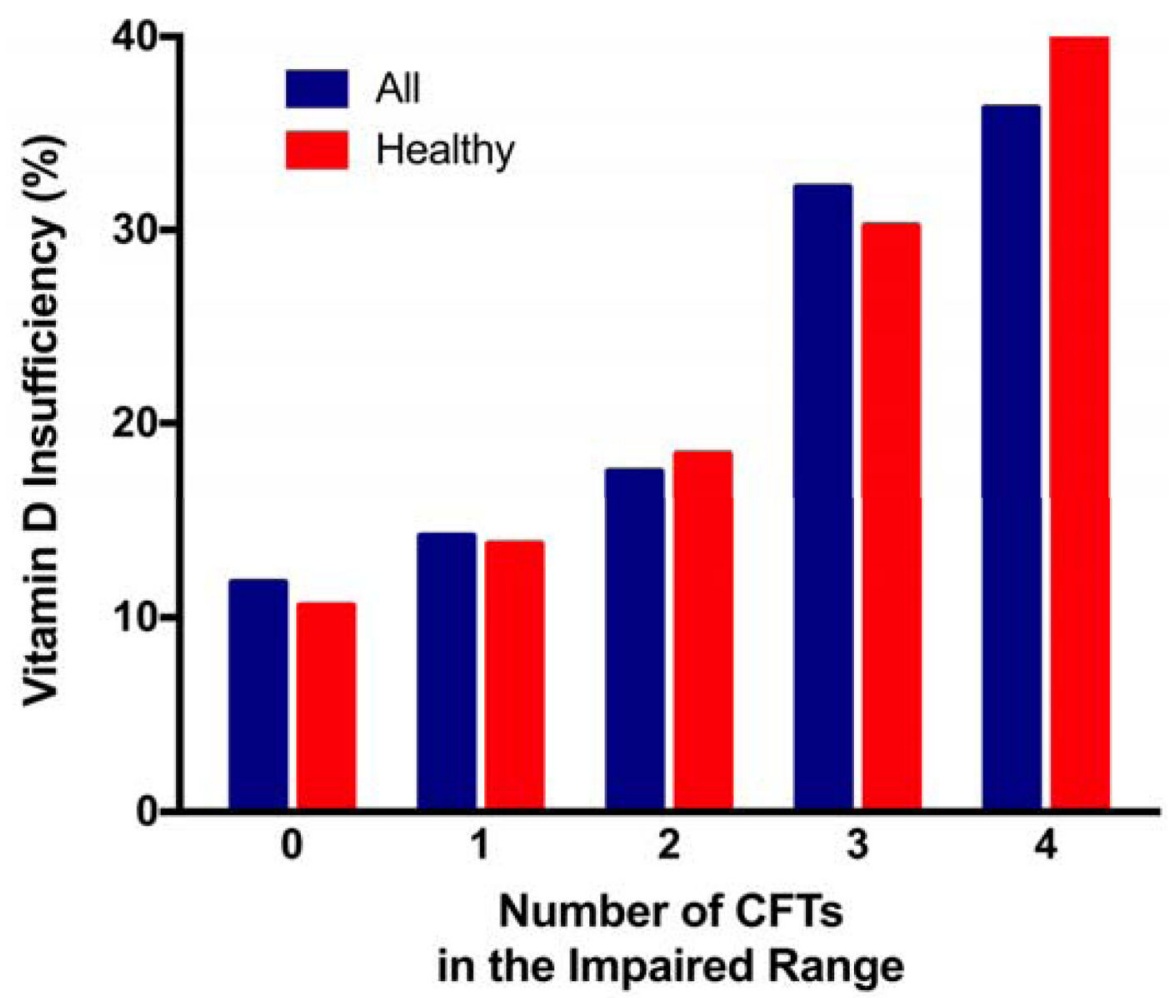

Figure 3.

Prevalence of VitD insufficiency by number of tests in the mildly cognitively impaired range for all participants (blue) and for the healthy subset (red). 


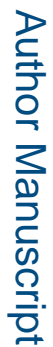

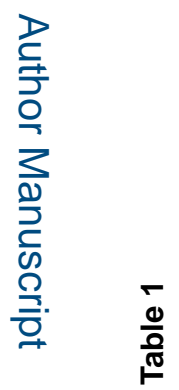

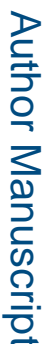

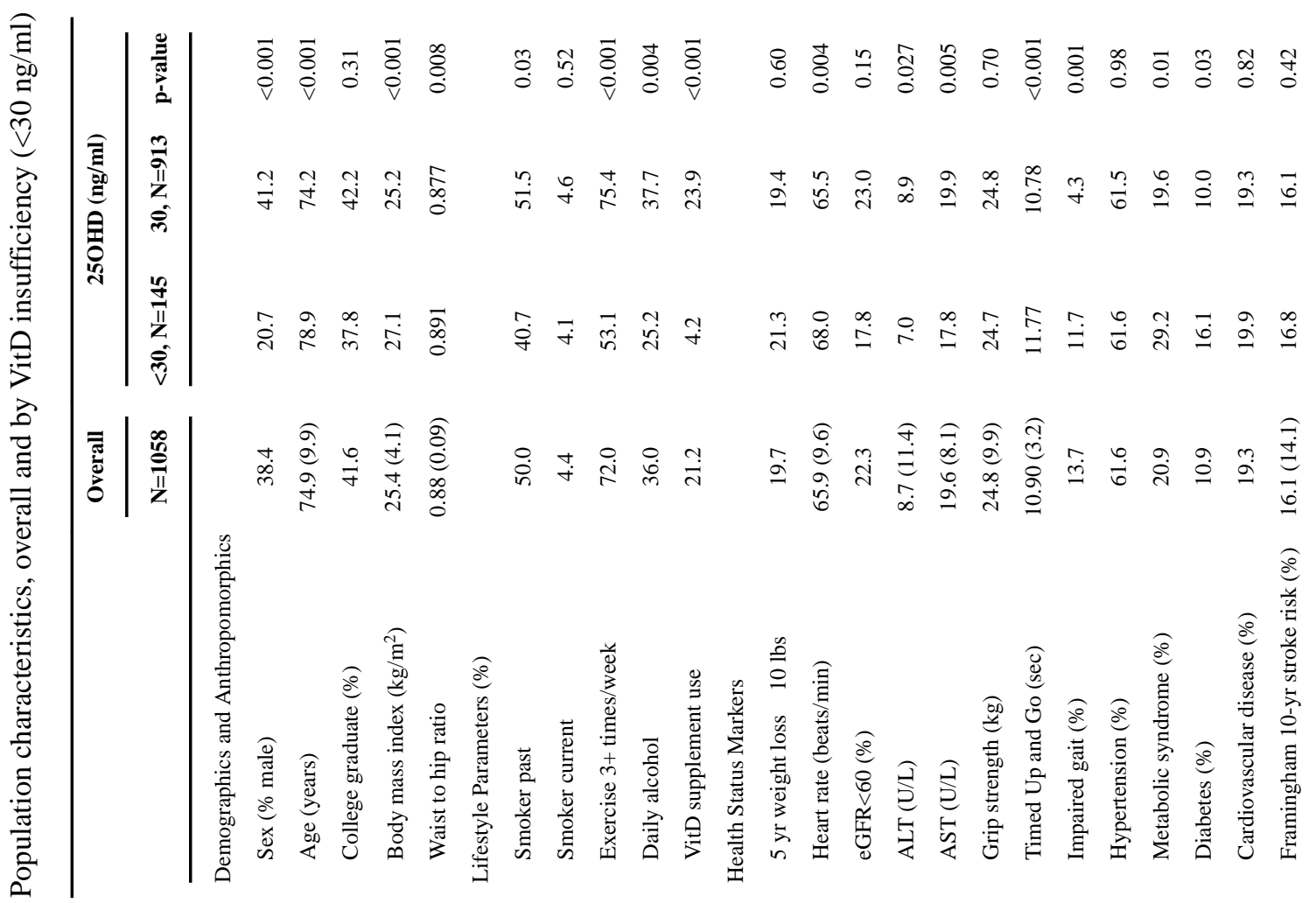

J Alzheimers Dis. Author manuscript; available in PMC 2018 May 16. 


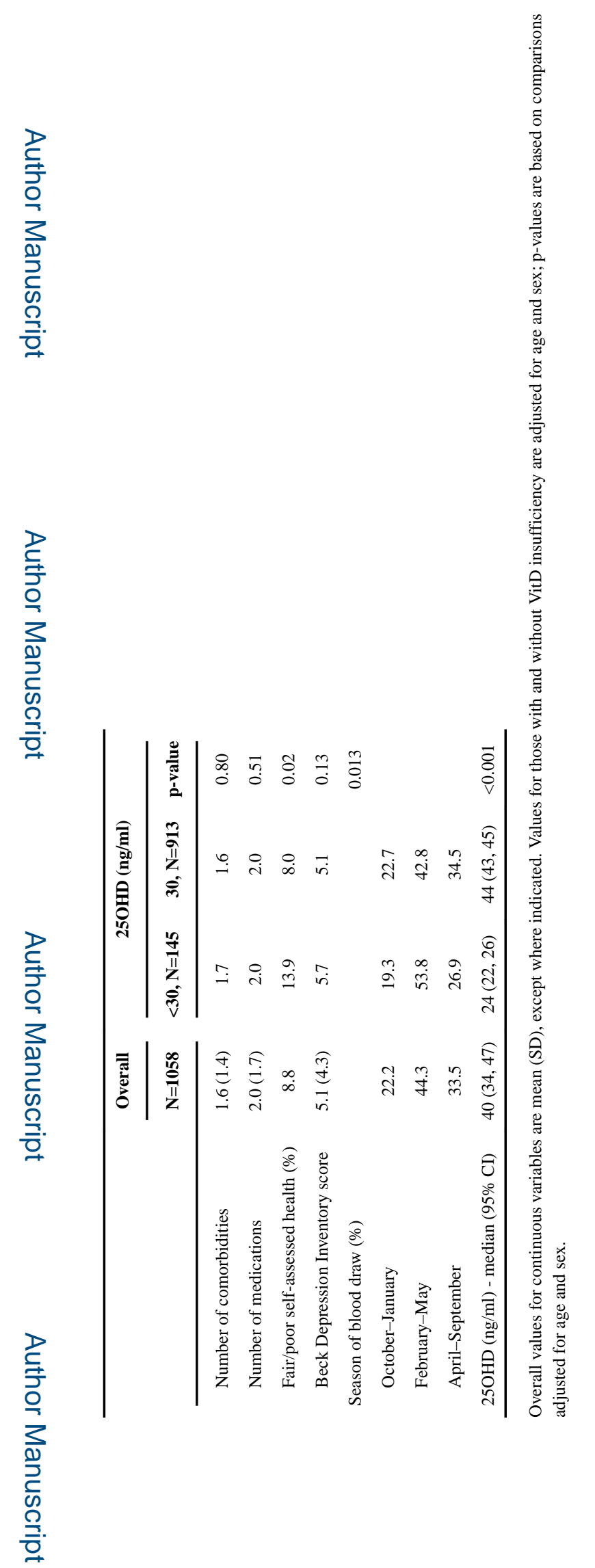




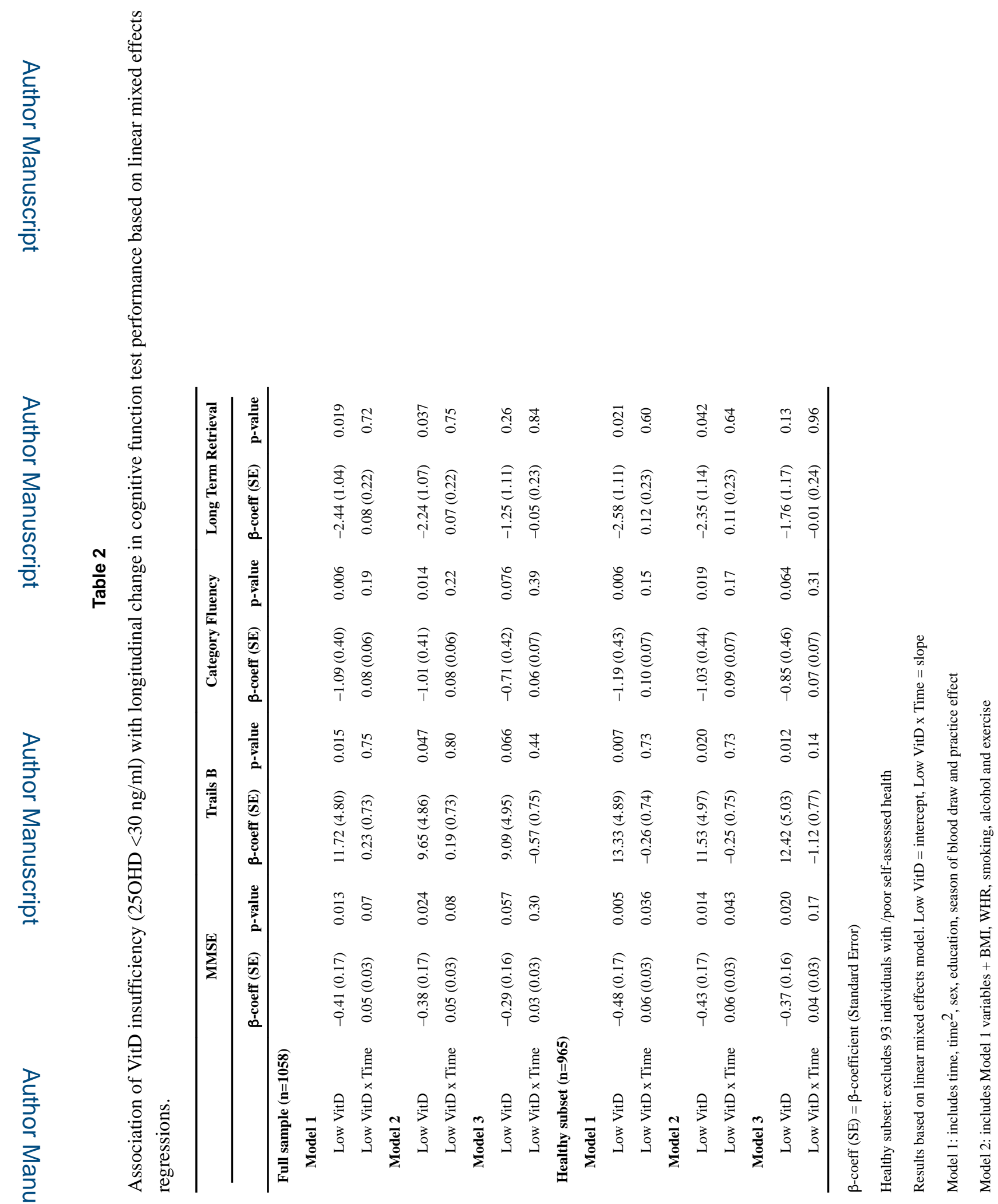




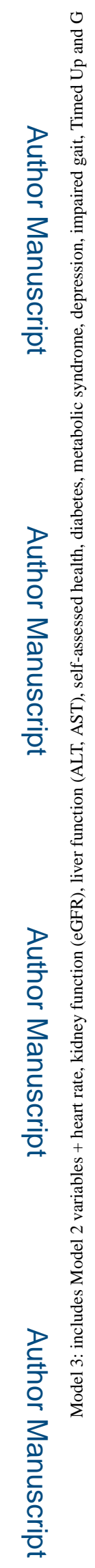




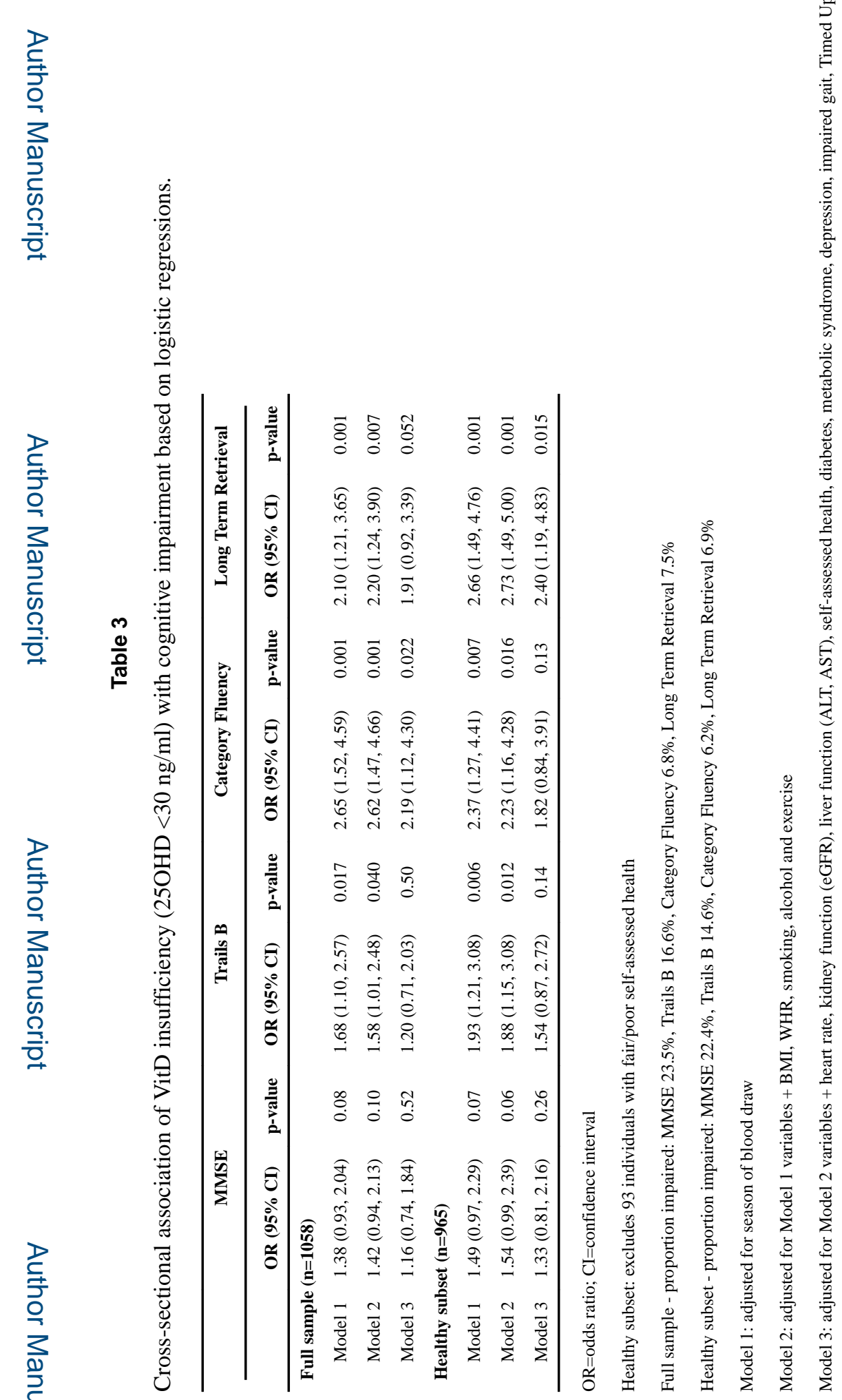




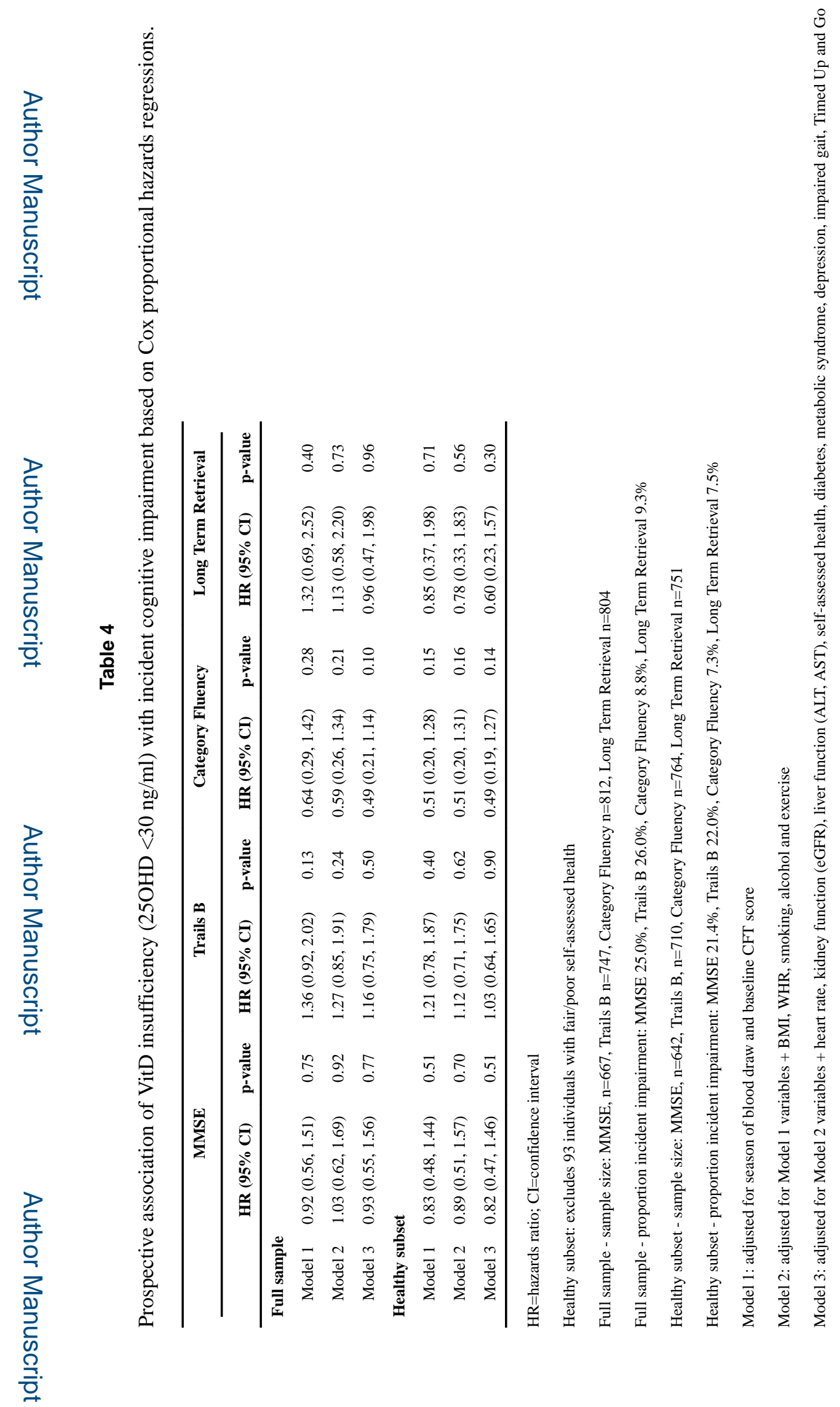

